\title{
EFFECT OF K-FELDSPAR ROCK FERTILIZER ON GROWTH AND MINERAL CONTENT OF MORINGA SEEDLINGS (MORINGA OLEIFERA)
}

\author{
A.I. Abou-Amer* and H.F. El-Tayeb ${ }^{* *}$ \\ * Soil Fert. \& Microbio. Res. Dept., Water Resources \& Desert Soils Div., D.R.C., Cairo, Egypt. \\ ** Botanical Gardens Res. Dept., Hort. Res. Inst. (Antoniades Br.), ARC, Alexandria, Egypt.
}

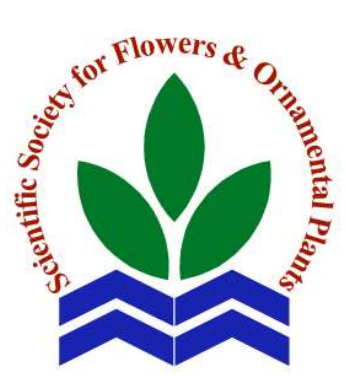

Scientific J. Flowers \& Ornamental Plants, 1(1):35-43 (2014).

Received:

$4 / 2 / 2014$

Revised by: Prof. Dr. M.G. El-Torky, Alexandria Univ.

Prof. Dr. Boshra A. ElSayed, Hort. Res. Inst., ARC.

ABSTRACT: A field experiment was conducted at the Botanical Gardens Research Department Land, Hort. Res. Inst. Alex., Egypt, during 2011-2012 and 2012-2013 seasons to study the effect of Kfeldspar rock fertilizer on growth and mineral content of Moringa seedlings. Feldspar grinded rock and potassium sulphate were used as fertilizer sources of $\mathrm{K}$ fertilizer to Moringa plants. Fertilizer treatments consisted of three rates of K-feldspar $(0,200,400 \mathrm{~g} /$ tree $)$ and three rates of $\mathrm{K}$-sulphate $(0,100,200 \mathrm{~g} /$ tree $)$, besides six combinations prepared from K-feldspar and K-sulphate. These amounts were added as a soil drench in two equal doses, the first one was applied immediately after planting (on April, $15^{\text {th. }}$ ), while the other after 3 months from the first (on July, $15^{\text {th. }}$ ). In addition, all Moringa seedlings received phosphate $\left(50 \mathrm{~g} \mathrm{P}_{2} \mathrm{O}_{5} /\right.$ tree $)$, organic manure $(3 \mathrm{~kg} /$ tree $)$ and $\mathrm{N}$ (50 g / tree) at processing and preparing the soil to planting seedlings. Also, in the second season, Moringa seedlings were cultivated at the same age and height as well as the same agricultural practices as in the first one.

The obtained results indicated that all treatments increased plant height and stem diameter with various significant differences compared to the control at the different determinations assessed in the two seasons. However, the superiority in the two seasons was for the combination of $200 \mathrm{~g} \mathrm{~K}-\mathrm{F}+100 \mathrm{~g} \mathrm{~K}-\mathrm{S}$, which gave the tallest plants and widest stems, as well as $\mathrm{K}$ mineral content of either soil or plant. In general, connecting between K-F and K-S recorded better results than the individual application of each in most determinations registered in both seasons. Also, increasing the rate of either K-F or K$\mathrm{S}$, individually or in combination caused an addition improvement in all previous characters measured in different stages of the two seasons. Moreover, treatments that involved K-feldspar gave better results in the second period of each season than that included K-sulphate alone, indicating its ability to provide plants with $\mathrm{K}^{+}$ions for a long period.

So, it can be recommended to fertilize Moringa seedlings with $200 \mathrm{~g} \mathrm{~K}$-feldspar grinded rock $+100 \mathrm{~g}$ of K-sulphate for to obtain the best growth with the least cost.

Key words: Potassium, feldspar rock, fertilizer, Moringa seedlings, growth, mineral content.

\section{INTRODUCTION}

Moringa oleifera Lam. belongs to the fam. of Moringaceae, is known as drumstick.
It is a medium-sized soft wood tree of about $10 \mathrm{~m}$ height. Leaves can be eaten fresh, coocked or stored as dry powder for many months without refrigeration, and reportedly 
without loss of nutritional value. Moringa is promising as a food source in the tropics as it becomes in full leaf at the end of dry season when other foods are typically scarce (Aiyelaagbe, 2011). The cakes of Moringa seeds increased the mineral content of the soil, and this in turn increased the yield of the maize crop compared to the control. So, Moringa has a significant economic importance because of its multiple-use, but it has been more intensely used in the industry, medicinal and in the feeding human and animal as protein source, Emmanuel et al. (2011).

Potassium (K) is one of the most important essential nutrients required for plant growth and production. In Egypt, hundred percent of potassium fertilizers are met only by imports, therefore, there is a great need to optimize the use of the natural resources to continue the development and sustainability of agriculture. The approach of applying mineral source of $\mathrm{K}$ from natural deposits has been introduced by many investigators to reduce the tremendous increase of chemical fertilizer costs, Rogers et al (1998). Chemical fertilizers, however have an adverse impact on environment plus their high cost which may hinder its use in developing countries. The alternative approach to reduce the tremendous increase of chemical fertilizer costs is to exploit indigenous resources such as K-bearing minerals. One of the most important Kminerals is $\mathrm{K}$-feldspar rock which its cost ranging between $600-800 \mathrm{LE} /$ ton against 7000-8000 LE/ton for the chemical fertilizer. However, $\mathrm{K}$ occurs in feldspar is very weathering-resistant framework lattice positions (Sanz and Rowell, 1988). So, the $\mathrm{K}^{+}$ion is not easily released and is therefore not suitable for direct application to the plants.

Little studies were done to reveal the effect of $\mathrm{K}$ rates or sources on the initial growth and on the partition and accumulation of this element in the different organs of Moringa plants. Among of them that was undertaken by Chaves et al. (2005) who mentioned that Moringa oleifera was not responsive to the $\mathrm{K}$ external concentration above $2 \mathrm{mM}$ as $\mathrm{KCl}$ salt. The $\mathrm{K}$ accumulation in stems was higher than in roots and leaves. The supplying with $2,4,6$, 8 and $12 \mathrm{mM}$ of $\mathrm{K}$ had not influenced on the translocation efficiency of $\mathrm{K}$ throughout the plants which showed to be more efficient to use $\mathrm{K}$ under a lower concentration of this ion in nutrient solution. Recently, Hussein and Abou-Baker (2014) found that all growth characters of Moringa plants increased with addition of potassium silicate and salicylic acid. On the other plants, Badr (2006) revealed that the response of tomato plants to the feldspar-compost (F-compost) inoculated with silicate dissolving bacteria (SDB) was dramatic when added to sandy soil of low K content and its effect was higher than Ksulphate. The conjunctive use of F-compost + SDB also produced maximum $\mathrm{K}$ use efficiency, total $\mathrm{K}$ uptake and considerable higher $\mathrm{K}$ recovery than $\mathrm{K}$-sulphate. Also, available $\mathrm{K}$ released from feldspar increased markedly through composting process and the maximum increase was observed with $40 \%$ feldspar addition. The benefit of $\mathrm{F}$ compost demonstrated the validity of sustained agronomic performance of tomato and reduces the cost of cultivation through the use of cheap feldspar. On the same line, were those results attained by Hellal et al. (2009) on faba bean, Labib et al. (2011) on cowpea, and Labib et al. (2012) on potato. In order to reduce the dependence on imported potash, feldspar a potash mineral, contains $11.25 \% \mathrm{~K}_{2} \mathrm{O}\left(\mathrm{KAlSi}_{3} \mathrm{O}_{8}\right)$ and therefore it could be a potential K-source for crop production. So, the main target of this trial is to evaluate the possibility of substituting partly or totally the expensive potassium fertilizer by natural deposits of feldspar bearing rocks.

\section{MATERIALS AND METHODS}

A field experiment was conducted at the Experimental Farm of the Botanical Gardens Dept., Hort. Res. Inst., Alex., Egypt, during the two consecutive seasons of 2011/12 and $2012 / 13$ to examine the effect of fertilization 
On the other hand, plant height $(\mathrm{cm})$ and basal stem diameter $(\mathrm{cm})$ were recorded at planting on April, $15^{\text {th }}$ and two times afterwards with 3 months interval, i.e. on July, $15^{\text {th }}$ and on October, $15^{\text {th }}$ in the two years of each season, whereas K content was determined in dry leaf samples as percentage using the method of A.O.A.C. (1980) and in soil samples taken randomly from the four main directions of the 3 replicates of each treatment at a depth of $0-30 \mathrm{~cm}$ as $\mathrm{mg} / \mathrm{kg}$ soil using the method described by Black et al. (1982) on July, $15^{\text {th }}$ and October, $15^{\text {th }}$ only of the two years of each season.

In the second season (2012-2013), Moringa seedlings were cultivated at the same age and height as well as the same agricultural practices as just done in the first season (2011-2012).
Data were then tabulated and statistically analyzed according to SAS institute program (1994) using Duncan's Multiple Range Test (Duncan, 1955) for elucidating the significancy between various treatments at $5 \%$ level.

\section{RESULTS AND DISCUSSION}

\section{Effect of potassium fertilization treatments on:}

\section{1- Plant height and stem diameter:}

It is clear from data averaged in Tables (1 and 2) that means of both plant height $(\mathrm{cm})$ and stem diameter at the base $(\mathrm{cm})$ were markedly improved in response to the different fertilization treatments applied in this study with various significant levels relative to control means at the different measuring times in the two seasons.

Table 1. Effect of potassium fertilization treatments on plant height $(\mathrm{cm})$ of Moringa oleifera Lam. plant during 2011/2012 and 2012/2013 seasons.

\begin{tabular}{|c|c|c|c|c|c|c|}
\hline \multirow{3}{*}{$\begin{array}{l}\text { Potassium } \\
\text { fertilization } \\
\text { treatments }\end{array}$} & \multicolumn{6}{|c|}{ First season: 2011-2012 } \\
\hline & \multicolumn{3}{|c|}{ First year: 2011} & \multicolumn{3}{|c|}{ Second year: 2012} \\
\hline & April, 15 ${ }^{\text {th }}$ & July, $15^{\text {th }}$ & October, $15^{\text {th }}$ & April, $15^{\text {th }}$ & July, $15^{\text {th }}$ & October, $15^{\text {th }}$ \\
\hline Control & $50.30 \mathrm{a}$ & $59.56 \mathrm{~h}$ & $78.30 \mathrm{f}$ & $109.35 \mathrm{~h}$ & $126.72 \mathrm{j}$ & $150.35 \mathrm{~g}$ \\
\hline $\mathrm{K}-\mathrm{F}$ at $200 \mathrm{~g}$ & $50.03 \mathrm{a}$ & $60.93 \mathrm{gh}$ & $82.48 \mathrm{e}$ & $112.94 \mathrm{gh}$ & $130.93 \mathrm{ij}$ & $157.00 \mathrm{f}$ \\
\hline K-F at $400 \mathrm{~g}$ & $49.36 \mathrm{a}$ & $62.03 \mathrm{~g}$ & $84.67 d$ & $113.99 \mathrm{~g}$ & $132.60 \mathrm{j}$ & $163.94 \mathrm{e}$ \\
\hline K-S at100g & $48.67 \mathrm{a}$ & $83.88 b$ & $105.53 \mathrm{bc}$ & $133.49 \mathrm{~d}$ & $143.22 \mathrm{~h}$ & $171.76 \mathrm{~cd}$ \\
\hline $\mathrm{K}-\mathrm{S}$ at $200 \mathrm{~g}$ & $49.25 \mathrm{a}$ & $88.70 \mathrm{a}$ & $109.80 \mathrm{a}$ & $135.45 \mathrm{c}$ & $151.93 \mathrm{f}$ & $173.75 \mathrm{c}$ \\
\hline $100 \mathrm{~g} \mathrm{K-F}+50 \mathrm{~g} \mathrm{K-S}$ & $49.20 \mathrm{a}$ & $73.82 d$ & $104.90 \mathrm{bc}$ & $130.36 \mathrm{e}$ & $148.91 \mathrm{~g}$ & $172.30 \mathrm{~cd}$ \\
\hline $200 \mathrm{~g} K-\mathrm{F}+100 \mathrm{~g} \mathrm{K-S}$ & $49.67 \mathrm{a}$ & $89.00 \mathrm{a}$ & $110.33 \mathrm{a}$ & $147.81 \mathrm{a}$ & $173.94 \mathrm{a}$ & $205.63 a$ \\
\hline $100 \mathrm{~g} K-\mathrm{F}+100 \mathrm{~g} \mathrm{K-S}$ & $48.54 \mathrm{a}$ & $85.08 \mathrm{~b}$ & $106.30 \mathrm{~b}$ & $141.93 b$ & $168.35 b$ & $188.82 b$ \\
\hline $200 \mathrm{~g} \mathrm{K-F}+50 \mathrm{~g} \mathrm{K-S}$ & $49.85 \mathrm{a}$ & $70.36 \mathrm{e}$ & $103.99 \mathrm{c}$ & $130.50 \mathrm{e}$ & $158.10 \mathrm{~d}$ & $171.33 \mathrm{~cd}$ \\
\hline $150 \mathrm{~g} \mathrm{K-F}+25 \mathrm{~g} \mathrm{K-S}$ & $50.00 \mathrm{a}$ & $65.33 \mathrm{f}$ & $103.16 \mathrm{c}$ & $126.73 f$ & $155.83 \mathrm{e}$ & $169.02 d$ \\
\hline $300 \mathrm{~g}$ K-F+50g K-S & $49.76 \mathrm{a}$ & $77.25 \mathrm{c}$ & $105.02 \mathrm{bc}$ & $135.03 \mathrm{c}$ & $162.56 \mathrm{c}$ & $185.86 b$ \\
\hline \multirow{2}{*}{$\begin{array}{l}\text { Potassium } \\
\text { fertilization } \\
\text { treatments }\end{array}$} & \multicolumn{6}{|c|}{ Second season: 2012-2013 } \\
\hline & \multicolumn{3}{|c|}{ First year: 2012} & \multicolumn{3}{|c|}{ Second year: 2013} \\
\hline Control & $54.33 \mathrm{a}$ & $64.26 \mathrm{~h}$ & $80.64 \mathrm{~g}$ & $103.48 \mathrm{i}$ & $125.35 \mathrm{j}$ & $150.00 \mathrm{f}$ \\
\hline $\mathrm{K}-\mathrm{F}$ at $200 \mathrm{~g}$ & $54.00 \mathrm{a}$ & $65.90 \mathrm{gh}$ & $89.10 \mathrm{f}$ & $120.00 \mathrm{~h}$ & $134.00 \mathrm{i}$ & $163.47 \mathrm{de}$ \\
\hline $\mathrm{K}-\mathrm{F}$ at $400 \mathrm{~g}$ & $53.50 \mathrm{a}$ & $67.28 \mathrm{~g}$ & $91.50 \mathrm{e}$ & $124.10 \mathrm{~g}$ & $142.73 \mathrm{~h}$ & $172.50 \mathrm{~cd}$ \\
\hline K-S at100g & $53.36 \mathrm{a}$ & $90.50 \mathrm{~b}$ & $105.76 \mathrm{~d}$ & $123.68 \mathrm{~g}$ & $150.19 \mathrm{~g}$ & $160.76 \mathrm{e}$ \\
\hline $\mathrm{K}-\mathrm{S}$ at $200 \mathrm{~g}$ & $53.20 \mathrm{a}$ & $95.80 \mathrm{a}$ & $112.86 \mathrm{cb}$ & $131.43 \mathrm{e}$ & $157.80 \mathrm{e}$ & $164.59 \mathrm{de}$ \\
\hline $100 \mathrm{~g} \mathrm{K-F}+50 \mathrm{~g} \mathrm{K-S}$ & $54.00 \mathrm{a}$ & $79.71 d$ & $113.30 \mathrm{bc}$ & $138.38 \mathrm{c}$ & $163.56 \mathrm{~cd}$ & $175.68 \mathrm{c}$ \\
\hline $200 \mathrm{~g} \mathrm{K-F}+100 \mathrm{~g} \mathrm{K-S}$ & $53.68 \mathrm{a}$ & $96.15 \mathrm{a}$ & $120.56 a$ & $156.76 \mathrm{a}$ & $187.92 \mathrm{a}$ & $210.76 a$ \\
\hline $100 \mathrm{~g} \mathrm{K-F}+100 \mathrm{~g} \mathrm{K-S}$ & $54.50 \mathrm{a}$ & $91.80 \mathrm{~b}$ & $114.00 \mathrm{~b}$ & $142.97 \mathrm{~b}$ & $169.69 b$ & $189.00 \mathrm{~b}$ \\
\hline $200 \mathrm{~g}$ K-F+50g K-S & $54.73 a$ & $76.33 \mathrm{e}$ & $112.32 \mathrm{bc}$ & $133.40 \mathrm{~d}$ & $160.33 d$ & $174.90 \mathrm{c}$ \\
\hline $150 \mathrm{~g} \mathrm{K-F}+25 \mathrm{~g} \mathrm{K-S}$ & $54.00 \mathrm{a}$ & $70.76 f$ & $111.24 \mathrm{c}$ & $129.08 \mathrm{f}$ & $152.91 \mathrm{f}$ & $166.33 d$ \\
\hline $300 \mathrm{~g} \mathrm{K-F}+50 \mathrm{~g} \mathrm{K-S}$ & $53.78 \mathrm{a}$ & $83.50 \mathrm{c}$ & $113.43 b$ & $138.20 \mathrm{c}$ & $165.00 \mathrm{c}$ & $190.00 \mathrm{~b}$ \\
\hline
\end{tabular}

* K-F: Potassium feldspar and K-S: Potassium sulphate.

* Means within a column having the same letters are not significantly different according to Duncan's Multiple Range Test at 5\% level. 
Table 2. Effect of potassium fertilization treatments on stem diameter (cm) of Moringa oleifera Lam. plant during 2011/2012 and 2012/2013 seasons.

\begin{tabular}{|c|c|c|c|c|c|c|}
\hline \multirow{3}{*}{$\begin{array}{l}\text { Potassium } \\
\text { fertilization } \\
\text { treatments }\end{array}$} & \multicolumn{6}{|c|}{ First season: 2011-2012 } \\
\hline & \multicolumn{3}{|c|}{ First year: 2011} & \multicolumn{3}{|c|}{ Second year: 2012} \\
\hline & April, 15 $^{\text {th }}$ & July, $15^{\text {th }}$ & October, $15^{\text {th }}$ & April, 15 $^{\text {th }}$ & July, $15^{\text {th }}$ & October, $15^{\text {th }}$ \\
\hline Control & $0.58 \mathrm{a}$ & $0.61 b$ & $0.88 \mathrm{f}$ & $1.10 \mathrm{f}$ & $1.39 \mathrm{e}$ & $1.70 \mathrm{e}$ \\
\hline K-F at 200g & $0.61 \mathrm{a}$ & $0.63 b$ & $0.91 \mathrm{ef}$ & $1.41 \mathrm{ed}$ & $1.71 \mathrm{~d}$ & $1.85 \mathrm{de}$ \\
\hline K-F at $400 \mathrm{~g}$ & $0.55 \mathrm{a}$ & $0.66 b$ & $0.99 \mathrm{e}$ & $1.50 \mathrm{~d}$ & $1.76 \mathrm{~d}$ & $1.99 \mathrm{~d}$ \\
\hline K-S at100g & $0.57 \mathrm{a}$ & $0.65 b$ & $1.11 \mathrm{~d}$ & $1.35 \mathrm{e}$ & $1.78 \mathrm{~d}$ & $2.04 \mathrm{~cd}$ \\
\hline $\mathrm{K}-\mathrm{S}$ at $200 \mathrm{~g}$ & $0.59 \mathrm{a}$ & $0.80 \mathrm{ba}$ & $1.15 \mathrm{c}$ & $1.50 \mathrm{~d}$ & $1.89 \mathrm{~cd}$ & $2.16 \mathrm{c}$ \\
\hline $100 \mathrm{~g} \mathrm{~K}-\mathrm{F}+50 \mathrm{~g} \mathrm{~K}-\mathrm{S}$ & $0.60 \mathrm{a}$ & $0.89 \mathrm{ab}$ & $1.15 \mathrm{c}$ & $1.65 \mathrm{c}$ & $2.01 \mathrm{bc}$ & $2.26 \mathrm{bc}$ \\
\hline $200 \mathrm{~g} \mathrm{K-F}+100 \mathrm{~g} \mathrm{K-S}$ & $0.60 \mathrm{a}$ & $1.08 \mathrm{a}$ & $1.50 \mathrm{a}$ & $2.07 \mathrm{a}$ & $2.40 \mathrm{a}$ & $2.50 \mathrm{a}$ \\
\hline $100 \mathrm{~g} \mathrm{K-F}+100 \mathrm{~g} \mathrm{K-S}$ & $0.56 \mathrm{a}$ & $1.00 \mathrm{a}$ & $1.21 \mathrm{~b}$ & $1.89 \mathrm{~b}$ & $2.18 \mathrm{~b}$ & $2.36 \mathrm{~b}$ \\
\hline $200 \mathrm{~g} \mathrm{K-F}+50 \mathrm{~g} \mathrm{K-S}$ & $0.58 \mathrm{a}$ & $0.80 \mathrm{ab}$ & $1.06 \mathrm{de}$ & $1.58 \mathrm{~cd}$ & $1.93 \mathrm{c}$ & $2.10 \mathrm{c}$ \\
\hline $150 \mathrm{~g} \mathrm{K-F}+25 \mathrm{~g} \mathrm{K-S}$ & $0.59 \mathrm{a}$ & $0.71 b$ & $1.10 \mathrm{~cd}$ & $1.50 \mathrm{~d}$ & $1.86 \mathrm{~cd}$ & $2.05 \mathrm{~cd}$ \\
\hline $300 \mathrm{~g} \mathrm{~K}-\mathrm{F}+50 \mathrm{~g} \mathrm{K-S}$ & $0.60 \mathrm{a}$ & $0.90 \mathrm{ab}$ & $1.10 \mathrm{~cd}$ & $1.70 \mathrm{c}$ & $2.00 \mathrm{cb}$ & $2.14 \mathrm{c}$ \\
\hline \multirow{2}{*}{$\begin{array}{l}\text { Potassium } \\
\text { fertilization } \\
\text { treatments }\end{array}$} & \multicolumn{6}{|c|}{ Second season: $2012-2013$} \\
\hline & \multicolumn{3}{|c|}{ First year: 2012} & \multicolumn{3}{|c|}{ Second year: 2013} \\
\hline Control & $0.58 \mathrm{a}$ & $0.60 \mathrm{c}$ & $0.79 \mathrm{f}$ & $1.07 \mathrm{~g}$ & $1.40 \mathrm{e}$ & $1.67 \mathrm{e}$ \\
\hline $\mathrm{K}-\mathrm{F}$ at $200 \mathrm{~g}$ & $0.60 \mathrm{a}$ & $0.63 c$ & $0.90 \mathrm{e}$ & $1.33 \mathrm{ef}$ & $1.64 \mathrm{~d}$ & $1.87 \mathrm{de}$ \\
\hline $\mathrm{K}-\mathrm{F}$ at $400 \mathrm{~g}$ & $0.59 \mathrm{a}$ & $0.70 \mathrm{bc}$ & $1.00 \mathrm{~d}$ & $1.50 \mathrm{~d}$ & $1.80 \mathrm{c}$ & $2.00 \mathrm{c}$ \\
\hline K-S at100g & $0.61 \mathrm{a}$ & $0.68 \mathrm{bc}$ & $0.92 \mathrm{de}$ & $1.28 \mathrm{f}$ & $1.70 \mathrm{~d}$ & $1.94 \mathrm{c}$ \\
\hline $\mathrm{K-S}$ at $200 \mathrm{~g}$ & $0.60 \mathrm{a}$ & $0.80 \mathrm{~b}$ & $1.00 \mathrm{~d}$ & $1.35 \mathrm{e}$ & $1.75 \mathrm{~cd}$ & $2.10 \mathrm{bc}$ \\
\hline $100 \mathrm{~g} \mathrm{K-F}+50 \mathrm{~g} \mathrm{K-S}$ & $0.61 \mathrm{a}$ & $0.90 \mathrm{ab}$ & $1.14 \mathrm{c}$ & $1.65 \mathrm{c}$ & $2.00 \mathrm{bc}$ & $2.20 \mathrm{bc}$ \\
\hline $200 \mathrm{~g} \mathrm{~K}-\mathrm{F}+100 \mathrm{~g} \mathrm{K-S}$ & $0.60 \mathrm{a}$ & $1.10 \mathrm{a}$ & $1.50 \mathrm{a}$ & $2.00 \mathrm{a}$ & $2.43 \mathrm{a}$ & $2.55 \mathrm{a}$ \\
\hline 100g K-F+100g K-S & $0.59 \mathrm{a}$ & $0.90 \mathrm{ab}$ & $1.26 \mathrm{~b}$ & $1.76 \mathrm{~b}$ & $2.10 \mathrm{~b}$ & $2.29 b$ \\
\hline $200 \mathrm{~g} \mathrm{K-F}+50 \mathrm{~g} \mathrm{K-S}$ & $0.61 \mathrm{a}$ & $0.83 \mathrm{ab}$ & $1.10 \mathrm{c}$ & $1.60 \mathrm{c}$ & $1.86 \mathrm{c}$ & $2.04 \mathrm{c}$ \\
\hline $150 \mathrm{~g} \mathrm{~K}-\mathrm{F}+25 \mathrm{~g} \mathrm{K-S}$ & $0.59 \mathrm{a}$ & $0.72 b c$ & $1.00 \mathrm{~d}$ & $1.39 \mathrm{e}$ & $1.75 \mathrm{~cd}$ & $1.96 \mathrm{c}$ \\
\hline $300 \mathrm{~g} \mathrm{~K}-\mathrm{F}+50 \mathrm{~g} \mathrm{K-S}$ & $0.61 \mathrm{a}$ & $0.88 \mathrm{ab}$ & $1.21 \mathrm{~b}$ & $1.70 \mathrm{~d}$ & $2.00 \mathrm{bc}$ & $2.10 \mathrm{bc}$ \\
\hline
\end{tabular}

* K-F: Potassium feldspar and K-S: Potassium sulphate.

* Means within a column having the same letters are not significantly different according to Duncan's Multiple Range Test at 5\% level.

However, the prevalence was for the combination between K-feldspar at $200 \mathrm{~g}$ and $\mathrm{K}$-sulphate at $100 \mathrm{~g}$ which gave the tallest and widest stem in the different stages of both seasons. This combination was followed by $100 \mathrm{~g} \mathrm{K-F}+100 \mathrm{~g}$ K-S combined treatment that recorded means occupied the second rank after the prevailing combination mentioned above. In general, connecting between K-feldspar and K-sulphate scored better results than the individual application of each in most determinations taken in both seasons. Also, increasing the rate of either $\mathrm{K}$-feldspar from 200 to $400 \mathrm{~g}$ /seedling or Ksulphate from 100 to $200 \mathrm{~g} / \mathrm{seedling}$, individually or in combination caused additional improvement in these two parameters in most cases of the first and second seasons. Moreover, treatments that involved K-feldspar at any level gave better results in the second period $\left(2^{\text {nd }}\right.$ year $)$ of each season than those included K-sulphate alone. This may be attributed to that K-feldspar usually acts as a slow-release fertilizer that supplies $\mathrm{K}^{+}$ions for a long period, while $\mathrm{K}$ sulphate is water-soluble-fertilizer and quickly lost with drainage water.

In this concern, Labib et al. (2011) stated that K-feldspar is quite resistant to weathering and supply relatively small quantities of $\mathrm{K}$ during the growing season. However, their cumulative release of $\mathrm{K}$ over several years is very important acting as slow-release fertilizer. The positive influence of the fine grains of the K-mineral bearing rocks is improving the poor structure of loose sandy soil, consequently the water and nutrient capacities of this soil will be 
enhanced and increase their ability to plant uptake. Labib et al. (2012) added that the excessive application of relatively soluble chemical fertilizers has hazardous impact on environmental conditions since considerable proportions are usually lost through drainage and cause pollution of water channels. Besides, the positive effects of K-feldspar not only ascribed to the multi-benefits of $\mathrm{K}^{+}$ ions, but also to its containing 70.4\% $\mathrm{SiO}_{2}$, $15.5 \% \quad \mathrm{Al}_{2} \mathrm{O}_{3}, 8.4 \% \mathrm{~K}_{2} \mathrm{O}, 3.2 \% \quad \mathrm{Na}_{2} \mathrm{O}$ and traces of other elements such as Fe, Mg, P and Ti (Labib et al., 2011).

The previous gains are in well agreement with those attained by Chaves et al. (2005) and Hussein and Abou-Baker (2014) on Moringa oleifera, Badr (2006) on tomato and Hellal et al. (2009) on faba bean.

\section{2- Potassium content in the leaves:}

Data in Table (3) exhibit that all potassium fertilization sources used in such trial, solely or in combination significantly increased $\mathrm{K}$ content $\%$ in the leaves of fertilized plants, with few exceptions when compared to the unfertilized ones at the different determinations undertaken during the two years of each season. Potassium sulphate alone recorded good content of $\mathrm{K}$ during the early stages of growth (the first year of each season), but this content was decreased afterwards. The opposite was the right concerning the effect of K-feldspar, as it raised $\mathrm{K}$ content slightly in the early stages, but caused a highly increment in the last stages (the second year of each season).

Table 3. Effect of potassium fertilization treatments on potassium (\%) in the dry leaves of Moringa oleifera Lam. plant during 2011/2012 and 2012/2013 seasons.

\begin{tabular}{|c|c|c|c|c|c|c|c|c|}
\hline \multirow{3}{*}{$\begin{array}{l}\text { Potassium } \\
\text { fertilization } \\
\text { treatments }\end{array}$} & \multicolumn{4}{|c|}{ First season: 2011-2012 } & \multicolumn{4}{|c|}{ Second season: $2012-2013$} \\
\hline & \multicolumn{2}{|c|}{ First year: 2011} & \multicolumn{2}{|c|}{ Second year: 2012} & \multicolumn{2}{|c|}{ First year: 2012} & \multicolumn{2}{|c|}{ Second year: 2013} \\
\hline & $\begin{array}{c}\text { July, } \\
15^{\text {th }}\end{array}$ & $\begin{array}{c}\text { October, } \\
15^{\text {th }}\end{array}$ & $\begin{array}{c}\text { July, } \\
15^{\text {th }}\end{array}$ & $\begin{array}{c}\text { October, } \\
15^{\text {th }} \\
\end{array}$ & $\begin{array}{c}\text { July, } \\
15^{\text {th }}\end{array}$ & $\begin{array}{c}\text { October, } \\
15^{\text {th }}\end{array}$ & $\begin{array}{c}\text { July, } \\
15^{\text {th }}\end{array}$ & $\begin{array}{c}\text { October, } \\
15^{\text {th }}\end{array}$ \\
\hline Control & $0.10 \mathrm{e}$ & $0.15 f$ & $0.21 \mathrm{e}$ & $0.26 \mathrm{e}$ & $0.12 \mathrm{e}$ & $0.17 \mathrm{e}$ & $0.24 \mathrm{e}$ & $0.23 \mathrm{f}$ \\
\hline K-F at $200 \mathrm{~g}$ & $0.11 \mathrm{e}$ & $0.20 \mathrm{e}$ & $0.32 \mathrm{de}$ & $0.49 \mathrm{~d}$ & $0.15 d$ & $0.25 \mathrm{~d}$ & $0.37 \mathrm{de}$ & $0.48 \mathrm{~d}$ \\
\hline $\mathrm{K}-\mathrm{F}$ at $400 \mathrm{~g}$ & $0.13 \mathrm{e}$ & $0.37 \mathrm{c}$ & $0.58 \mathrm{~b}$ & $0.86 b$ & $0.18 \mathrm{~cd}$ & $0.36 \mathrm{c}$ & $0.60 \mathrm{~b}$ & $0.80 \mathrm{~b}$ \\
\hline K-S at100g & $0.20 \mathrm{c}$ & $0.34 \mathrm{c}$ & $0.36 \mathrm{~d}$ & $0.34 \mathrm{de}$ & $0.22 b$ & $0.38 b c$ & $0.40 \mathrm{~d}$ & $0.36 \mathrm{e}$ \\
\hline $\mathrm{K}-\mathrm{S}$ at $200 \mathrm{~g}$ & $0.27 \mathrm{~b}$ & $0.40 \mathrm{~b}$ & $0.37 \mathrm{~d}$ & $0.38 \mathrm{~d}$ & $0.30 \mathrm{ab}$ & $0.44 b$ & $0.41 \mathrm{~d}$ & $0.36 \mathrm{e}$ \\
\hline $100 \mathrm{~g} \mathrm{~K}-\mathrm{F}+50 \mathrm{~g} \mathrm{K-S}$ & $0.18 \mathrm{~d}$ & $0.30 \mathrm{~d}$ & $0.55 b$ & $0.65 \mathrm{c}$ & $0.20 \mathrm{c}$ & $0.33 c$ & $0.48 \mathrm{c}$ & $0.63 \mathrm{c}$ \\
\hline $200 \mathrm{~g} K-\mathrm{F}+100 \mathrm{~g} \mathrm{K-S}$ & $0.34 \mathrm{a}$ & $0.56 \mathrm{a}$ & $0.69 \mathrm{a}$ & $0.91 \mathrm{a}$ & $0.36 \mathrm{a}$ & $0.60 \mathrm{a}$ & $0.71 \mathrm{a}$ & $0.96 \mathrm{a}$ \\
\hline $100 \mathrm{~g} \mathrm{~K}-\mathrm{F}+100 \mathrm{~g} \mathrm{~K}-\mathrm{S}$ & $0.28 \mathrm{~b}$ & $0.37 \mathrm{c}$ & $0.50 \mathrm{c}$ & $0.69 \mathrm{c}$ & $0.30 \mathrm{ab}$ & $0.41 b$ & $0.50 \mathrm{c}$ & $0.67 \mathrm{bc}$ \\
\hline $200 \mathrm{~g} \mathrm{~K}-\mathrm{F}+50 \mathrm{~g} \mathrm{K-S}$ & $0.18 \mathrm{~d}$ & $0.33 \mathrm{c}$ & $0.54 \mathrm{bc}$ & $0.71 \mathrm{c}$ & $0.21 b c$ & $0.33 c$ & $0.58 \mathrm{~b}$ & $0.73 b c$ \\
\hline $150 \mathrm{~g} \mathrm{~K}-\mathrm{F}+25 \mathrm{~g} \mathrm{~K}-\mathrm{S}$ & $0.16 \mathrm{de}$ & $0.28 \mathrm{de}$ & $0.50 \mathrm{c}$ & $0.63 \mathrm{~cd}$ & $0.18 \mathrm{~cd}$ & $0.30 \mathrm{~cd}$ & $0.47 \mathrm{c}$ & $0.61 \mathrm{c}$ \\
\hline $300 \mathrm{~g} \mathrm{~K}-\mathrm{F}+50 \mathrm{~g} \mathrm{K-S}$ & $0.20 \mathrm{c}$ & $0.39 b c$ & $0.56 \mathrm{~b}$ & $0.81 b$ & $0.25 \mathrm{~b}$ & $0.41 \mathrm{~b}$ & $0.55 \mathrm{bc}$ & $0.80 \mathrm{~b}$ \\
\hline
\end{tabular}

* K-F: Potassium feldspar and K-S: Potassium sulphate.

* Means within a column having the same letters are not significantly different according to Duncan's Multiple Range Test at 5\% level.

This could be reasonable because of the quick soluble of $\mathrm{K}$-sulphate in water and lossing, while $\mathrm{K}$-feldspar slowly releases $\mathrm{K}^{+}$ ions for a long-term period. Joining between $\mathrm{K}$-feldspar and $\mathrm{K}$-sulphate induced more beneficial effect than the individual applying of each one, with the mastery of $200 \mathrm{~g} \mathrm{k}$ feldspar $+100 \mathrm{~g}$ K-sulphate combined treatment that recorded the utmost high $\mathrm{K}$ content over that of control at the various determinations registered in the two seasons.
The aforementioned results can be interpreted and discussed as done before in case of plant height and stem diameter traits. However, analogous observations were also detected by Badr (2006) on tomato, Labib et al. (2011) on cowpea and Labib et al. (2012), who noted that addition of equal rates of K-feldspar and K-sulphate resulted in the highest content of starch, monosurose, protein and both vitamin $\mathrm{A}$ and $\mathrm{C}$ in potato tubers. In this connection, Hussein and Abou-Baker (2014) revealed that K-silicate 
in combination with salicylic acid gave the higher increases in $\mathrm{N}, \mathrm{P}$ and $\mathrm{K}$ in the leaves of Moringa plants.

\section{3- Potassium content in the soil:}

As shown in Table (4), potassium content in the soil $(\mathrm{mg} / \mathrm{kg}$ soil) amended with the different sources of $\mathrm{K}$ at various rates took a parallel trend to that of $\mathrm{K}$ content in the leaves of Moringa plants treated with the same sources and rates. So, this content was higher in the soil at the two determinations menstruated in the first year of both seasons due to the sole application of K-sulphate at the high level $(200 \mathrm{~g} /$ seedling). That was true in the second year of both seasons when K-feldspar was added individually at the high rate $(400 \mathrm{~g} / \mathrm{seedling})$.

Table 4. Effect of potassium fertilization treatments on potassium content $(\mathrm{mg} / \mathrm{kg})$ in the soil cultivated with Moringa oleifera Lam. plant during 2011/2012 and 2012/2013 seasons.

\begin{tabular}{|c|c|c|c|c|c|c|c|c|}
\hline \multirow{3}{*}{$\begin{array}{l}\text { Potassium } \\
\text { fertilization } \\
\text { treatments }\end{array}$} & \multicolumn{4}{|c|}{ First season: 2011-2012 } & \multicolumn{4}{|c|}{ Second season: $2012-2013$} \\
\hline & \multicolumn{2}{|c|}{ First year: 2011} & \multicolumn{2}{|c|}{ Second year: 2012} & \multicolumn{2}{|c|}{ First year: 2012} & \multicolumn{2}{|c|}{ Second year: 2013} \\
\hline & July, $15^{\text {th }}$ & $\begin{array}{c}\text { October, } \\
15^{\text {th }}\end{array}$ & July, $15^{\text {th }}$ & $\begin{array}{c}\text { October, } \\
15^{\text {th }}\end{array}$ & July, $15^{\text {th }}$ & $\begin{array}{c}\text { October, } \\
15^{\text {th }} \\
\end{array}$ & July, $15^{\text {th }}$ & $\begin{array}{c}\text { October, } \\
15^{\text {th }}\end{array}$ \\
\hline Control & $43.89 \mathrm{~h}$ & $41.90 \mathrm{~g}$ & $38.70 \mathrm{~g}$ & $30.81 \mathrm{f}$ & $48.27 \mathrm{~h}$ & $46.00 \mathrm{~g}$ & $40.58 \mathrm{~h}$ & $33.69 \mathrm{~h}$ \\
\hline $\mathrm{K}-\mathrm{F}$ at $200 \mathrm{~g}$ & $46.51 \mathrm{~g}$ & $48.62 f$ & $56.90 \mathrm{e}$ & $59.33 \mathrm{~cd}$ & $51.70 \mathrm{~g}$ & $53.50 \mathrm{f}$ & $62.70 \mathrm{e}$ & $65.30 \mathrm{~d}$ \\
\hline $\mathrm{K}-\mathrm{F}$ at $400 \mathrm{~g}$ & $48.50 \mathrm{f}$ & $58.20 \mathrm{c}$ & $66.71 b$ & $71.30 \mathrm{ab}$ & $53.50 f$ & $61.70 \mathrm{bc}$ & $73.40 \mathrm{~b}$ & $74.67 b$ \\
\hline K-S at100g & $60.36 b$ & $58.42 \mathrm{c}$ & $51.39 \mathrm{f}$ & $40.63 \mathrm{e}$ & $58.76 \mathrm{de}$ & $56.33 \mathrm{e}$ & $50.00 \mathrm{~g}$ & $40.00 \mathrm{~g}$ \\
\hline $\mathrm{K}-\mathrm{S}$ at $200 \mathrm{~g}$ & $62.31 \mathrm{a}$ & $60.11 \mathrm{~b}$ & $56.50 \mathrm{e}$ & $48.90 \mathrm{~d}$ & $64.71 b$ & $61.50 \mathrm{bc}$ & $55.47 f$ & $46.63 f$ \\
\hline $100 \mathrm{~g} \mathrm{~K}-\mathrm{F}+50 \mathrm{~g} \mathrm{~K}-\mathrm{S}$ & $53.42 d$ & $55.32 \mathrm{de}$ & $57.85 \mathrm{de}$ & $58.85 \mathrm{c}$ & $58.68 \mathrm{de}$ & $61.00 \mathrm{ba}$ & $64.70 d$ & $66.32 \mathrm{~cd}$ \\
\hline $200 \mathrm{~g} \mathrm{~K}-\mathrm{F}+100 \mathrm{~g} \mathrm{~K}-\mathrm{S}$ & $62.48 \mathrm{a}$ & $69.00 \mathrm{a}$ & $70.12 \mathrm{a}$ & $72.46 \mathrm{a}$ & $67.92 \mathrm{a}$ & $75.90 \mathrm{a}$ & $76.98 \mathrm{a}$ & $80.31 \mathrm{a}$ \\
\hline $100 \mathrm{~g} \mathrm{~K}-\mathrm{F}+100 \mathrm{~g} \mathrm{~K}-\mathrm{S}$ & $56.63 \mathrm{c}$ & $58.26 \mathrm{c}$ & $60.00 \mathrm{c}$ & $62.70 \mathrm{~b}$ & $62.30 \mathrm{c}$ & $63.80 \mathrm{~b}$ & $65.79 \mathrm{~cd}$ & $68.20 \mathrm{c}$ \\
\hline $200 \mathrm{~g} \mathrm{~K}-\mathrm{F}+50 \mathrm{~g} \mathrm{~K}-\mathrm{S}$ & $52.40 \mathrm{e}$ & $55.00 \mathrm{de}$ & $58.76 \mathrm{~d}$ & $60.00 \mathrm{~b}$ & $56.58 \mathrm{e}$ & $60.50 \mathrm{c}$ & $62.71 \mathrm{e}$ & $67.00 \mathrm{~cd}$ \\
\hline $150 \mathrm{~g} \mathrm{~K}-\mathrm{F}+25 \mathrm{~g} \mathrm{~K}-\mathrm{S}$ & $50.96 \mathrm{ef}$ & $63.00 \mathrm{e}$ & $55.63 \mathrm{fe}$ & $58.29 \mathrm{c}$ & $55.00 \mathrm{ef}$ & $58.46 \mathrm{~d}$ & $61.20 \mathrm{ef}$ & $62.33 \mathrm{e}$ \\
\hline $300 \mathrm{~g} \mathrm{~K}-\mathrm{F}+50 \mathrm{~g} \mathrm{~K}-\mathrm{S}$ & $54.00 \mathrm{~d}$ & $56.93 \mathrm{~d}$ & $59.90 \mathrm{~cd}$ & $60.36 \mathrm{~b}$ & $59.41 \mathrm{~d}$ & $61.60 \mathrm{bc}$ & $66.00 \mathrm{c}$ & $68.49 \mathrm{c}$ \\
\hline
\end{tabular}

* K-F: Potassium feldspar and K-S: Potassium sulphate.

* Means within a column having the same letters are not significantly different according to Duncan's Multiple Range Test at $5 \%$ level.

However, such content reached the maximum at combining between $200 \mathrm{~g} \mathrm{~K}$ feldspar and $100 \mathrm{~g} \mathrm{~K}$-sulphate, where this combination registered the highest $\mathrm{K}$ content in the soil at the different stages throughout the two seasons. The presence of K-feldspar, alone or in combined treatment continuously provides the soil with $\mathrm{K}^{+}$ions at various stages over many years, as it acts as a slowrelease fertilizer as mentioned before. The advantages of applying K-bearing rock on poor fertility sandy soil can be related to their improvement of physical and chemical properties, particularly when combined with organic amendment (Labib et al., 2012). Manning (2010) claimed that under conditions where soils are rapidly leached with low capacity to retain soluble nutrients, the use of K-feldspar does give a yield response greater than conventional fertilizers.
On the same line, were those results postulated by Badr (2006) on tomato, Labib et al. (2011) on cowpea and Labib et al. (2012) on potato.

From the previous findings, it can be concluded that binding between K-feldspar at the rate of $200 \mathrm{~g} / \mathrm{seedling}$ and $\mathrm{K}$-sulphate at the rate of $100 \mathrm{~g} / \mathrm{seedling}$ is the best combination for the best growth of Moringa seedlings cultivated in loamy sand soil and for reducing the cost of cultivation through the use of cheap feldspar.

\section{ACKNOWLEDGEMENT}

We thank Prof. Dr. Sayed M. Shahin, Head Researches Emeritus of Botanical Gardens. Res. Dept., Hort. Res. Inst., ARC for his helpful suggestions during preparing this manuscript. 


\section{REFERENCES}

Aiyelaagbe, I.O. (2011). Nigerian Hort.: Facing the Challenges of Human Health and Agricultural Productivity. Keynote address presented at the $29^{\text {th }}$ Annual National Conf. of Hort. Soci. of Nigeria (HORTSON): 24-29 July.

A.O.A.C. (1980). Official Methods of Analysis of the Association Official Agricultural Chemists. $15^{\text {th }} \quad$ Ed., Arlington, Virginia 22201:877-878.

Badr, M.A. (2006). Efficiency of K-feldspar combined with organic materials and silicate dissolving bacteria on tomato yield. J. Appli. Sci. Res., 2(12):11911198.

Black，C.A.; Evans， D.D.; White, J.I.; Ensminger, L.E. and Clark, F.E. (1982). Methods of Soil Analysis. Amer. Soc. Agronomy. Inc. Publisher Madison, Wisconsin. U.S.A.

Chaves, L.H.; R.A. Viegas; C.F. de Vasconcelos, Ana and H. Vieira (2005). Effect of potassium on moringa plants in nutrient solution. Revista de Biologia E Ciencias Daterra, 5(2):19-28.

Das, M.N. and Giri, N.C. (1986). Design and Analysis Experiments, $2^{\text {nd }}$ Ed., Puplished by Mohinder Singh Sejwal for Wiley, New Delhi 110 002, 488 pp.

Duncan, D.B. (1955). Multiple range and multiple F-tests. J. Biometrics, 11:1-42.

Emmanuel, S.A.; Zaku, S.G.; Adedirin, S.O.; Muazu, Tafida and Thomas, S.A. (2011). Moringa oleifera seed-cake, alternative biodegradable and biocompatibility organic fertilizer for modern farming. Agric. \& Biob. J. N. Amer., 2(9):12891292.
Hellal, F.A.; Abd El-Hady, M. and Ragab, A.A.M. (2009). Influence of organic amendment on nutrient availability and uptake by faba bean plants fertilized by rock phosphate and feldspar. AmericanEurasian J. Agric. \& Environ. Sci., 6(3):271-279.

Hussein, M.M. and Nesreen, H. Abou-Baker (2014). Growth and mineral status of moringa plants as affected by silicate and salicylic acid under salt stress. Inter. J. of Plant \& soil Sci., 3(2):163-177.

Labib B.F.; Ghabour, Th. K.; Rahim, I.S. and Wahba, M.M (2012). Effect of potassium bearing rock on the growth and quality of potato crop (Solanum tuberosum). Inter. J. of Res. in Management, 2(2):108-115.

Labib, B.F., Ghabour, Th. K., Wahba, M. M. and Rahim, I.S. (2011) Application of Kbearing rock for fertilization of cowpea (Vigna unguiculata). Inter. Res. J. of Geology \& Mining, 1(2):27-33.

Manning, D.A.C. (2010). Mineral sources of potassium for plant nutrition. A Review Agron. Sustain, Dev., pp. 281-294.

Richards, L.A. (1954). Diagnosis and Improvement Saline and Alkali Soils. USDA, Handbook No.60.

Rogers, J.R.; Bennett, P.C.; Choi, W.J. (1998). Felspars as a source of nutrients for microorganisms. Amer. Mineralogist., 83:1532-1540.

Sanz-Sconvino, J.J. and Rowell, D.L. (1988). The use of feldspar as potassium fertilizers in the Savannah of Columbia. Fertilizer Research, 17:71-83.

SAS Institute (1994). SAS/STAT User's Guide, Statistics, Vers. 6.04, $4^{\text {th }}$ Ed., SAS Institute Inc., Cary, N.C., USA. 


\section{تأثير التسميد البوتاسى بصخر الفلسبار على النمو والمحتوى المعدني لشتلات المورينجا}

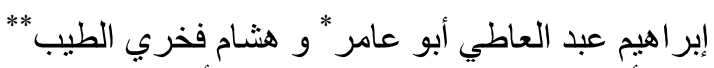

* قسم خصوبة و ميكروبيولوجيا الأراضي، شعبة مصادر المياه و الأر اضي الصحر الهية الطية، مركز بحوث الصحر اء،

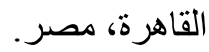

** قسم بحوث الحدائق النباتية، معهد بحوث البساتين (فرع أنطونيادس)، الأسكندرية، مصر.

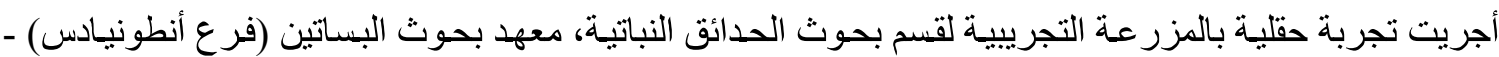

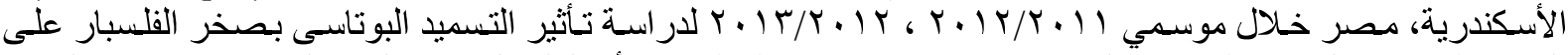

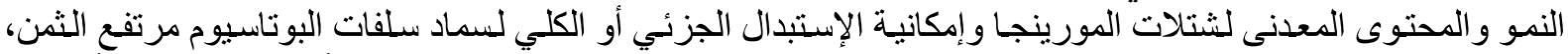

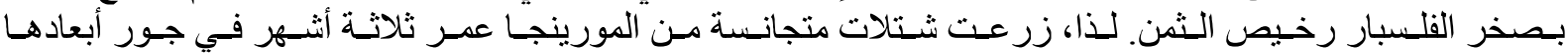

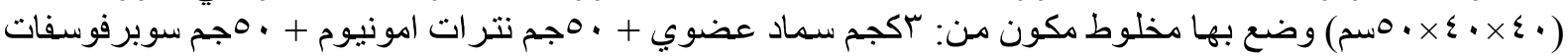

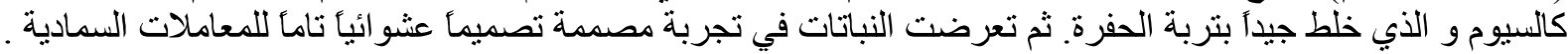

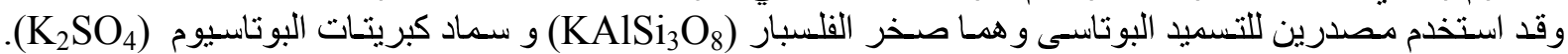

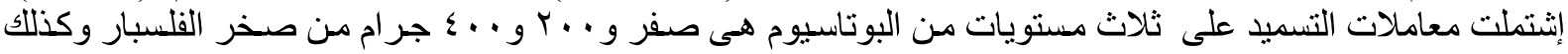

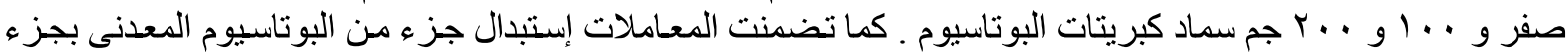

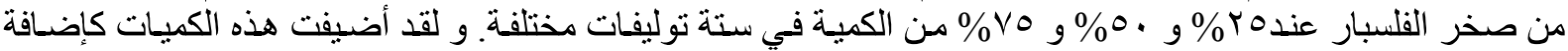

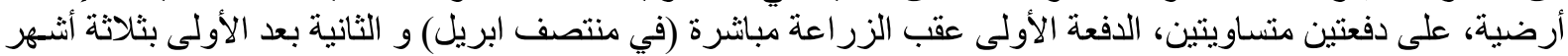

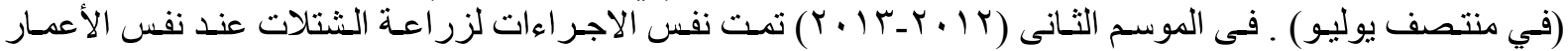

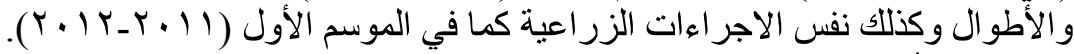

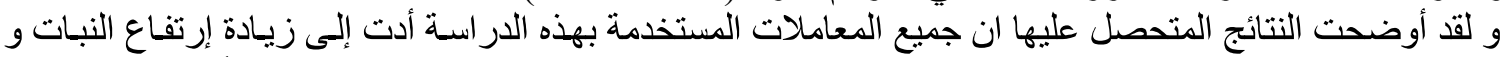

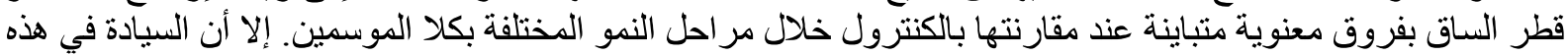

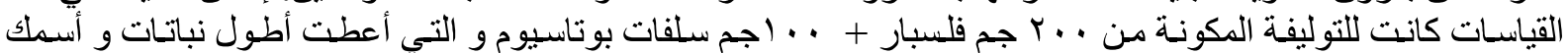

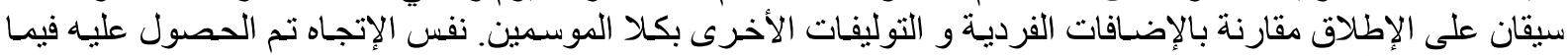

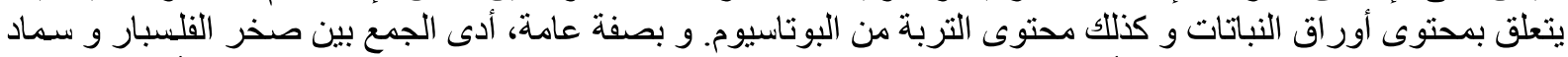

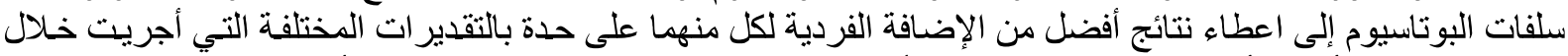

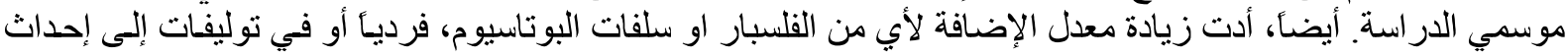

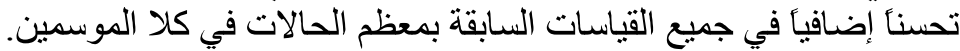

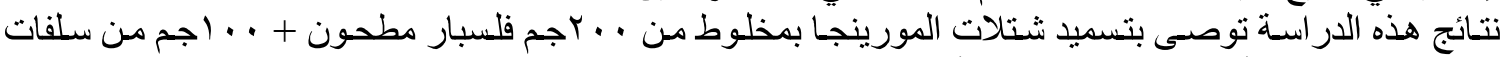
البوتاسيوم للحصول عذه الفي أفضل نمو للثتنات وبأقل تكلفة ممكنة. 